Real Analysis Exchange

Vol. 23(2), 1997-1998, pp. 689-695

In Soo Baek*, Pusan University of Foreign Studies, Pusan 608-738, Korea

e-mail: isbaek@taejo.pufs.ac.kr

\title{
DIMENSIONS OF WEAKLY CONVERGENT DERANGED CANTOR SETS
}

\begin{abstract}
We calculate the Hausdorff and packing dimensions of a generalized Cantor set which satisfies some condition on contracting ratios of its construction stages.
\end{abstract}

\section{Introduction}

In [2] we investigated the Hausdorff and packing dimensions of a deranged Cantor set whose contracting ratios and gap ratios in its construction are random but uniformly bounded away from 0. We used Frostman's density theorems in this study. In this paper, we consider a weakly convergent deranged Cantor set, which satisfies a condition that all the sequences of the solutions of some power equations related to the contracting ratios in its construction converge to some number. We note that in this case we deal with a deranged Cantor set having a weaker condition (a condition of a local uniform boundedness) than that of uniform boundedness of contracting ratios and gap ratios. We prove that its Hausdorff and packing dimensions are equal to the number and find the dimensions of the product of these kinds of sets. We finish with concrete examples of such deranged Cantor sets of positive and finite Hausdorff and packing measure of the corresponding dimension.

\section{Preliminaries}

We defined deranged Cantor set [2]. Let $I_{\phi}=[0,1]$. We obtain the left subinterval $I_{\tau, 1}$ and the right subinterval $I_{\tau, 2}$ of $I_{\tau}$ by deleting the middle open subinterval of $I_{\tau}$ inductively for each $\tau \in\{1,2\}^{n}$, where $n=0,1,2, \cdots$. Consider $E_{n}=\cup_{\tau \in\{1,2\}^{n}} I_{\tau}$. Then $\left\{E_{n}\right\}$ is a decreasing sequence of closed

\footnotetext{
Mathematical Reviews subject classification: 28A80

Received by the editors November 24,1997

* The author wishes to acknowledge the financial support of the Korea Research Foundation made in the program year of 1997
} 
sets. For each $n$, we put $\left|I_{\tau, 1}\right| /\left|I_{\tau}\right|=c_{\tau, 1}$ and $\left|I_{\tau, 2}\right| /\left|I_{\tau}\right|=c_{\tau, 2}$ for all $\tau \in\{1,2\}^{n}$, where $|I|$ denotes the diameter of $I$. Then $F=\bigcap_{n=0}^{\infty} E_{n}$ is a deranged Cantor set. We note that if $c_{\tau, 1}=a_{n+1}$ and $c_{\tau, 2}=b_{n+1}$ for all $\tau \in\{1,2\}^{n}$ for each $n$, then $F=\bigcap_{n=0}^{\infty} E_{n}$ is called a perturbed Cantor set [1].

We recall the $s$-dimensional Hausdorff measure of $F$ is defined by $H^{s}(F)=$ $\lim _{\delta \rightarrow 0} H_{\delta}^{s}(F)$ where

$$
H_{\delta}^{s}(F)=\inf \left\{\sum_{n=1}^{\infty}\left|U_{n}\right|^{s}:\left\{U_{n}\right\}_{n=1}^{\infty} \text { is a } \delta \text { - cover of } F\right\}
$$

and the Hausdorff dimension of $F$ is defined by

$$
\operatorname{dim}_{H}(F)=\sup \left\{s>0: H^{s}(F)=\infty\right\}\left(=\inf \left\{s>0: H^{s}(F)=0\right\}\right)(\text { see [3] }) .
$$

Also we recall that the $s$-dimensional packing measure of $F$ is defined by

$$
p^{s}(F)=\inf \left\{\sum_{n=1}^{\infty} P^{s}\left(F_{n}\right): \bigcup_{n=1}^{\infty} F_{n}=F\right\}
$$

where $P^{s}(E)=\lim _{\delta \rightarrow 0} P_{\delta}^{s}(E)$ and $P_{\delta}^{s}(E)=\sup \left\{\sum_{n=1}^{\infty}\left|U_{n}\right|^{s}:\left\{U_{n}\right\}\right.$ is a $\delta$-packing of $E\}$, and the packing dimension of $F$ :

$$
\operatorname{dim}_{p}(F)=\sup \left\{s>0: p^{s}(F)=\infty\right\}\left(=\inf \left\{s>0: p^{s}(F)=0\right\}\right)([3]) .
$$

We note that if $\left\{a_{n}\right\}$ and $\left\{b_{n}\right\}$ are given, then a perturbed Cantor set $F$ is determined. If numbers $c_{\tau}$ are given, then a deranged Cantor set is determined. We also note that a perturbed Cantor set is a special example of a deranged Cantor set. But in this paper we consider a specific class of deranged Cantor sets which is essentially different from a perturbed Cantor set. We are now ready to study the ratio geometry of the deranged Cantor set.

\section{Main results}

In this section, $F$ denotes a deranged Cantor set determined by $\left\{c_{\tau}\right\}$ with $\tau \in\{1,2\}^{n}$ where $n=1,2, \cdots$. Hereafter we only consider a deranged Cantor set whose contracting ratios $c_{\tau}$ and gap ratios $d_{\tau}\left(=1-\left(c_{\tau, 1}+c_{\tau, 2}\right)\right)$ are locally uniformly bounded away from 0 in the sense that for each $\sigma \in\{1,2\}^{N}$, $\left\{c_{\sigma \mid k}\right\}_{k=1}^{\infty}$ and $\left\{d_{\sigma \mid k}\right\}_{k=1}^{\infty}$ are uniformly bounded away from 0 . We note that if $x \in F$, then there is $\sigma \in\{1,2\}^{N}$ such that $\bigcap_{k=0}^{\infty} I_{\sigma \mid k}=\{x\}$. (Here $\sigma \mid k=$ $i_{1}, i_{2}, \cdots, i_{k}$ where $\sigma=i_{1}, i_{2}, \cdots, i_{k}, i_{k+1}, \cdots$.) 
Theorem 1. Let $c_{\tau} \in(0,1)$. Let $s_{\tau}$ be the solution to the equation $c_{\tau, 1}^{s_{\tau}}+c_{\tau, 2}^{s_{\tau}}=$ 1 for each $\tau$ of finite length. Suppose that $s_{\sigma \mid k}$ converges to $s$ as $k \rightarrow \infty$ for each $\sigma \in\{1,2\}^{N}$ ( $\sigma$ corresponds to $\left.x \in F\right)$. Then $\operatorname{dim}_{H}(F)=\operatorname{dim}_{p}(F)=s$.

Proof. We may assume that for each $\sigma \in\{1,2\}^{N}, c_{\sigma \mid k}, d_{\sigma \mid k}\left(=1-\left(c_{\sigma \mid k, 1}+\right.\right.$ $\left.\left.c_{\sigma \mid k, 2}\right)\right)>\alpha_{\sigma}$ for all integers $k$ for some small $\alpha_{\sigma}>0$. (In fact, it is redundant to assume that $c_{\sigma \mid k}>\alpha_{\sigma}$ for all integers $k$ for some small $\alpha_{\sigma}>0$ since $\left\{s_{\sigma \mid k}\right\}$ converges to $s$ and $d_{\sigma \mid k}>\alpha_{\sigma}>0$ for all $k$. For, if not, $\left\{s_{\sigma \mid k}\right\}$ doesn't converge to $s$.) Let $x \in F$. Then there is $\sigma \in\{1,2\}^{N}$ such that $\bigcap_{k=0}^{\infty} I_{\sigma \mid k}=\{x\}$. Let

$$
E_{n}(m)=\bigcap_{k \geq n}\left\{x \in F: \bigcap_{k=0}^{\infty} I_{\sigma \mid k}=\{x\}, s_{\sigma \mid k}>s-\frac{1}{m}\right\} .
$$

Now we only need to show that $\operatorname{dim}_{H}\left(E_{n}(m)\right) \geq s-\frac{2}{m}$ for each $m$ to have $\operatorname{dim}_{H}(F) \geq s$. Fix $m$ and let $\sigma \in\{1,2\}^{N}$. Then $c_{\sigma \mid k, 1}^{s_{\sigma \mid k}}+c_{\sigma \mid k, 2}^{s_{\sigma \mid k}}=1$ for each $k$. If $s_{\sigma \mid k}>s-\frac{1}{m}$, then $c_{\sigma \mid k, 1}^{s-\frac{2}{m}}+c_{\sigma \mid k, 2}^{s-\frac{2}{m}}>a>1$ for some $a>1$. Then

$$
\liminf _{k \rightarrow \infty}\left(c_{1}^{s-\frac{2}{m}}+c_{2}^{s-\frac{2}{m}}\right)\left(c_{\sigma \mid 1,1}^{s-\frac{2}{m}}+c_{\sigma \mid 1,2}^{s-\frac{2}{m}}\right) \cdots\left(c_{\sigma \mid k, 1}^{s-\frac{2}{m}}+c_{\sigma \mid k, 2}^{s-\frac{2}{m}}\right)=\infty
$$

for each $x \in E_{n}(m)$ with $\bigcap_{k=0}^{\infty} I_{\sigma \mid k}=\{x\}$.

We define a set function $\mu$ by

$$
\mu\left(I_{\tau}\right)=\frac{\left|I_{\tau}\right|^{s-\frac{2}{m}}}{\left(c_{1}^{s-\frac{2}{m}}+c_{2}^{s-\frac{2}{m}}\right)\left(c_{i_{1}, 1}^{s-\frac{2}{m}}+c_{i_{1}, 2}^{s-\frac{2}{m}}\right) \cdots\left(c_{i_{1}, i_{2}, \cdots, i_{k-1}, 1}^{s-\frac{2}{m}}+c_{i_{1}, i_{2}, \cdots, i_{k-1}, 2}^{s-\frac{2}{m}}\right)}
$$

for each $\tau=i_{1}, i_{2}, \cdots, i_{k-1}, i_{k}$, where $i_{j} \in\{1,2\}$. Clearly $\mu\left(I_{\tau}\right)=\mu\left(I_{\tau, 1}\right)+$ $\mu\left(I_{\tau, 2}\right)$ for all $\tau$ of finite length. Then $\mu$ can be extended to a mass distribution on $F$. (See [3,Proposition 1.7].) Given a small positive number $r$, there exists $k$ such that $\left|I_{\sigma \mid k+1}\right| \leq r<\left|I_{\sigma \mid k}\right|$. Since $d_{\sigma \mid j}\left|I_{\sigma \mid j}\right| \geq \alpha_{\sigma}\left|I_{\sigma \mid k}\right|>\alpha_{\sigma} r$ for $0 \leq j \leq k$, $B_{\alpha_{\sigma} r}(x) \subset\left[\bigcup_{\tau(\neq \sigma \mid k) \in\{1,2\}^{k}} I_{\tau}\right]^{c}$, where $B_{\alpha_{\sigma} r}(x)$ is the ball of radius $\alpha_{\sigma} r$ with center $x$. Thus $\mu\left(B_{\alpha_{\sigma} r}(x)\right) \leq \mu\left(I_{\sigma \mid k}\right)$. Now,

$$
\begin{aligned}
\frac{\mu\left(B_{\alpha_{\sigma} r}(x)\right)}{\left(\alpha_{\sigma} r\right)^{s-\frac{2}{m}}} & \leq \frac{\mu\left(I_{\sigma \mid k}\right)}{\alpha_{\sigma}^{s-\frac{2}{m}}\left|I_{\sigma \mid k+1}\right|^{s-\frac{2}{m}}} \\
& \leq \frac{\mu\left(I_{\sigma \mid k}\right)}{\alpha_{\sigma}^{s-\frac{2}{m}}\left(\alpha_{\sigma}^{s-\frac{2}{m}}\left|I_{\sigma \mid k}\right|^{s-\frac{2}{m}}\right)} \\
& =\frac{\left|I_{\sigma \mid k}\right|^{s-\frac{2}{m}}}{\alpha_{\sigma}^{2\left(s-\frac{2}{m}\right)}\left|I_{\sigma \mid k}\right|^{s-\frac{2}{m}}\left(c_{1}^{s-\frac{2}{m}}+c_{2}^{s-\frac{2}{m}}\right) \cdots\left(c_{\sigma \mid k-1,1}^{s-\frac{2}{m}}+c_{\sigma \mid k-1,2}^{s-\frac{2}{m}}\right)}
\end{aligned}
$$




$$
=\frac{1}{\alpha_{\sigma}^{2\left(s-\frac{2}{m}\right)}\left(c_{1}^{s-\frac{2}{m}}+c_{2}^{s-\frac{2}{m}}\right)\left(c_{\sigma \mid 1,1}^{s-\frac{2}{m}}+c_{\sigma \mid 1,2}^{s-\frac{2}{m}}\right) \cdots\left(c_{\sigma \mid k-1,1}^{s-\frac{2}{m}}+c_{\sigma \mid k-1,2}^{s-\frac{2}{m}}\right)}
$$

Then for $x \in E_{n}(m)$,

$$
\limsup _{r \rightarrow 0} \frac{\mu\left(B_{r}(x)\right)}{r^{s-\frac{2}{m}}} \leq \limsup _{k \rightarrow \infty} \frac{1}{\alpha_{\sigma}^{2\left(s-\frac{2}{m}\right)}\left(c_{1}^{s-\frac{2}{m}}+c_{2}^{s-\frac{2}{m}}\right) \cdots\left(c_{\sigma \mid k, 1}^{s-\frac{2}{m}}+c_{\sigma \mid k, 2}^{s-\frac{2}{m}}\right)}=0 .
$$

There exists a large number $n$ such that $\mu\left(E_{n}(m)\right)>0$ since $E_{n}(m)$ is a Borel set in $[0,1]$ and $E_{n}(m) \uparrow F$ as $n \uparrow \infty$ with $\mu(F)=1$. Thus $H^{s-\frac{2}{m}}(F)=\infty$ by Proposition 4.9 [3]. Hence $\operatorname{dim}_{H}(F) \geq s-\frac{2}{m}$.

Similarly we need only show that $\operatorname{dim}_{p}\left(E_{n}(m)\right) \leq s+\frac{2}{m}$ for each $m$ to have $\operatorname{dim}_{p}(F) \leq s$. Fix $m$ and let $\sigma \in\{1,2\}^{N}$. Then $c_{\sigma \mid k, 1}^{s_{\sigma \mid k}}+c_{\sigma \mid k, 2}^{s_{\sigma \mid k}}=1$ for each $k$. If $s_{\sigma \mid k}<s+\frac{1}{m}$, then $c_{\sigma \mid k, 1}^{s+\frac{2}{m}}+c_{\sigma \mid k, 2}^{s+\frac{2}{m}}<b<1$ for some $0<b<1$. Then

$$
\limsup _{k \rightarrow \infty}\left(c_{1}^{s+\frac{2}{m}}+c_{2}^{s+\frac{2}{m}}\right)\left(c_{\sigma \mid 1,1}^{s+\frac{2}{m}}+c_{\sigma \mid 1,2}^{s+\frac{2}{m}}\right) \cdots\left(c_{\sigma \mid k, 1}^{s+\frac{2}{m}}+c_{\sigma \mid k, 2}^{s+\frac{2}{m}}\right)=0
$$

for each $x \in G_{n}(m)$ with $\bigcap_{k=0}^{\infty} I_{\sigma \mid k}=\{x\}$, where

$$
G_{n}(m)=\bigcap_{k \geq n}\left\{x \in F: \bigcap_{k=0}^{\infty} I_{\sigma \mid k}=\{x\}, s_{\sigma \mid k}<s+\frac{1}{m}\right\}
$$

We define a set function $\nu$ by

$$
\nu\left(I_{\tau}\right)=\frac{\left|I_{\tau}\right|^{s+\frac{2}{m}}}{\left(c_{1}^{s+\frac{2}{m}}+c_{2}^{s+\frac{2}{m}}\right)\left(c_{i_{1}, 1}^{s+\frac{2}{m}}+c_{i_{1}, 2}^{s+\frac{2}{m}}\right) \cdots\left(c_{i_{1}, i_{2}, \cdots, i_{k-1}, 1}^{s+\frac{2}{m}}+c_{i_{1}, i_{2}, \cdots, i_{k-1}, 2}^{s+\frac{2}{m}}\right)}
$$

for each $\tau=i_{1}, i_{2}, \cdots, i_{k-1}, i_{k}$, where $i_{j} \in\{1,2\}$. Clearly $\nu\left(I_{\tau}\right)=\nu\left(I_{\tau, 1}\right)+$ $\nu\left(I_{\tau, 2}\right)$ for all $\tau$ of finite length. Then $\nu$ can be extended to a mass distribution on $F$. (See [3,Proposition 1.7].) Given a small positive number $r$, there exists $k$ such that $\left|I_{\sigma \mid k+1}\right| \leq r<\left|I_{\sigma \mid k}\right|$. Then $\frac{\nu\left(B_{r}(x)\right)}{r^{s+\frac{2}{m}}} \geq \frac{\nu\left(I_{\sigma \mid k+1}\right)}{\left|I_{\sigma \mid k}\right|^{s+\frac{2}{m}}}$. Since 


$$
\begin{aligned}
& \left|I_{\sigma \mid k+1}\right| /\left|I_{\sigma \mid k}\right|=c_{\sigma \mid k+1}>\alpha_{\sigma} \text { for all } k, \\
& \frac{\nu\left(B_{r}(x)\right)}{r^{s+\frac{2}{m}}} \geq \frac{\nu\left(I_{\sigma \mid k+1}\right)}{\left(\frac{1}{\alpha_{\sigma}}\right)^{s+\frac{2}{m}}\left|I_{\sigma \mid k+1}\right|^{s+\frac{2}{m}}} \geq \frac{\alpha_{\sigma}^{s+\frac{2}{m}} \nu\left(I_{\sigma \mid k+1}\right)}{\left|I_{\sigma \mid k+1}\right|^{s+\frac{2}{m}}} \\
& =\frac{\alpha_{\sigma}^{s+\frac{2}{m}}\left|I_{\sigma \mid k+1}\right|^{s+\frac{2}{m}}}{\left|I_{\sigma \mid k+1}\right|^{s+\frac{2}{m}}\left(c_{1}^{s+\frac{2}{m}}+c_{2}^{s+\frac{2}{m}}\right)\left(c_{\sigma \mid 1,1}^{s+\frac{2}{m}}+c_{\sigma \mid 1,2}^{s+\frac{2}{m}}\right) \cdots\left(c_{\sigma \mid k, 1}^{s+\frac{2}{m}}+c_{\sigma \mid k, 2}^{s+\frac{2}{m}}\right)} \\
& =\frac{\alpha_{\sigma}^{s+\frac{2}{m}}}{\left(c_{1}^{s+\frac{2}{m}}+c_{2}^{s+\frac{2}{m}}\right)\left(c_{\sigma \mid 1,1}^{s+\frac{2}{m}}+c_{\sigma \mid 1,2}^{s+\frac{2}{m}}\right) \cdots\left(c_{\sigma \mid k, 1}^{s+\frac{2}{m}}+c_{\sigma \mid k, 2}^{s+\frac{2}{m}}\right)}
\end{aligned}
$$

Then for $x \in G_{n}(m)$,

$$
\liminf _{r \rightarrow 0} \frac{\nu\left(B_{r}(x)\right)}{r^{s+\frac{2}{m}}} \geq \liminf _{k \rightarrow \infty} \frac{\alpha_{\sigma}^{s+\frac{2}{m}}}{\left(c_{1}^{s+\frac{2}{m}}+c_{2}^{s+\frac{2}{m}}\right) \cdots\left(c_{\sigma \mid k, 1}^{s+\frac{2}{m}}+c_{\sigma \mid k, 2}^{s+\frac{2}{m}}\right)}=\infty .
$$

Thus $p^{s+\frac{2}{m}}\left(G_{n}(m)\right)=0$ by Proposition 2.2 [4]. Since $p^{s+\frac{2}{m}}\left(G_{n}(m)\right)=0$ for each $n$ and $G_{n}(m)$ is a Borel set in $[0,1]$ and $G_{n}(m) \uparrow F$ as $n \uparrow \infty, p^{s+\frac{2}{m}}(F)=0$. Hence $\operatorname{dim}_{p}(F) \leq s+\frac{2}{m}$.

Remark. If we consider two covering functions

$h^{s}(F)=\liminf _{k \rightarrow \infty} \min _{\sigma \in\{1,2\}^{k}}\left(c_{1}^{s}+c_{2}^{s}\right)\left(c_{\sigma \mid 1,1}^{s}+c_{\sigma \mid 1,2}^{s}\right)\left(c_{\sigma \mid 2,1}^{s}+c_{\sigma \mid 2,2}^{s}\right) \cdots\left(c_{\sigma \mid k, 1}^{s}+c_{\sigma \mid k, 2}^{s}\right)$

and

$Q^{s}(F)=\limsup _{k \rightarrow \infty} \max _{\sigma \in\{1,2\}^{k}}\left(c_{1}^{s}+c_{2}^{s}\right)\left(c_{\sigma \mid 1,1}^{s}+c_{\sigma \mid 1,2}^{s}\right)\left(c_{\sigma \mid 2,1}^{s}+c_{\sigma \mid 2,2}^{s}\right) \cdots\left(c_{\sigma \mid k, 1}^{s}+c_{\sigma \mid k, 2}^{s}\right)$,

we can get some information [2] concerning the Hausdorff and packing dimensions of a deranged Cantor set using the covering dimensions generated by the above two covering functions respectively. First, if we define the lower Cantor dimension and the upper Cantor dimension of a deranged Cantor set $F$ by $\operatorname{dim}_{\underline{C}}(F)=\sup \left\{s>0: h^{s}(F)=\infty\right\}$ and $\operatorname{dim}_{\bar{C}}(F)=\sup \left\{s>0: Q^{s}(F)=\right.$ $\infty\}$, we see that

$$
0<\liminf _{n \rightarrow \infty} s_{n} \leq \operatorname{dim}_{\underline{C}}(F) \leq \operatorname{dim}_{\bar{C}}(F) \leq \limsup _{n \rightarrow \infty} S_{n}<1,
$$

where $s_{n}=\min _{\sigma \in\{1,2\}^{n}} s_{\sigma}, S_{n}=\max _{\sigma \in\{1,2\}^{n}} s_{\sigma}$ and $s_{\sigma}$ is the solution of the equation $c_{\sigma, 1}^{s_{\sigma}}+c_{\sigma, 2}^{s_{\sigma}}=1$. We note that there are many examples which 
satisfy the assumption of Theorem 1 essentially but still have gaps between $\liminf _{n \rightarrow \infty} s_{n}$ and $\limsup _{n \rightarrow \infty} S_{n}$. However we observe that in a perturbed Cantor set [1] for each $n s_{\sigma}=s_{n}=S_{n}$ for all $\sigma \in\{1,2\}^{n}$.

Second, we see that $\operatorname{dim}_{\underline{C}}(F) \leq \operatorname{dim}_{H}(F) \leq \operatorname{dim}_{p}(F) \leq \operatorname{dim}_{\bar{C}}(F)$ using arguments similar to those used in the proof of Theorem 1 . Similarly we have the result that if $0<h^{s}(F) \leq Q^{s}(F)<\infty$, then $0<H^{s}(F) \leq p^{s}(F)<\infty$.

Corollary 2. In a deranged Cantor set $A$, let $s_{\tau}$ be the solution of the equation $c_{\tau, 1}^{s_{\tau}}+c_{\tau, 2}^{s_{\tau}}=1$ for each $\tau$ of finite length. Suppose that $s_{\sigma \mid k}$ converges to $s$ as $k \rightarrow \infty$ for each $\sigma \in\{1,2\}^{N}$. In a deranged Cantor set $B$, let $t_{\tau}$ be the solution of the equation $c_{\tau, 1}^{t_{\tau}}+c_{\tau, 2}^{t_{\tau}}=1$ for each $\tau$ of finite length. Suppose that $t_{\sigma \mid k}$ converges to $t$ as $k \rightarrow \infty$ for each $\sigma \in\{1,2\}^{N}$. Then $\operatorname{dim}_{H}(A \times B)=$ $\operatorname{dim}_{p}(A \times B)=s+t$.

Proof. It follows immediately from the fact [5] that if $\operatorname{dim}_{H}(A)=\operatorname{dim}_{p}(A)$ and $\operatorname{dim}_{H}(B)=\operatorname{dim}_{p}(B)$, then $\operatorname{dim}_{H}(A \times B)=\operatorname{dim}_{p}(A \times B)=\operatorname{dim}_{H}(A)+$ $\operatorname{dim}_{H}(B)$.

Example 3. There is an alternating, strongly convergent deranged Cantor set having positive and finite s-dimensional Hausdorff and packing measure where $s$ is the limit of the sequence of solutions of the power equations. (To be precise, for each $\sigma \in\{1,2\}^{N},\left\{s_{\sigma \mid k}-s\right\}_{k}^{\infty}$ alternates and $\left|s_{\sigma \mid k}-s\right| \downarrow 0$.)

Proof. It is easy to show that there exist $0<B_{1}<B_{2}<\infty$ such that

$$
B_{1}<\left(c_{1}^{s}+c_{2}^{s}\right)\left(c_{\sigma \mid 1,1}^{s}+c_{\sigma \mid 1,2}^{s}\right)\left(c_{\sigma \mid 2,1}^{s}+c_{\sigma \mid 2,2}^{s}\right) \cdots\left(c_{\sigma \mid k, 1}^{s}+c_{\sigma \mid k, 2}^{s}\right)<B_{2}
$$

for all $\sigma$ and $k$. In the proof of Theorem 1, using the above facts, for all $x \in F$ we get

$$
0<\frac{\alpha_{\sigma}^{s}}{B_{2}} \leq \liminf _{r \rightarrow 0} \frac{\lambda\left(B_{r}(x)\right)}{r^{s}} \leq \limsup _{r \rightarrow 0} \frac{\lambda\left(B_{r}(x)\right)}{r^{s}} \leq \frac{1}{\alpha_{\sigma}^{2 s} B_{1}}<\infty
$$

where $\lambda$ is the Borel measure on $F$ generated from the set function

$$
\lambda\left(I_{\tau}\right)=\frac{\left|I_{\tau}\right|^{s}}{\left(c_{1}^{s}+c_{2}^{s}\right)\left(c_{i_{1}, 1}^{s}+c_{i_{1}, 2}^{s}\right) \cdots\left(c_{i_{1}, i_{2}, \cdots, i_{k-1}, 1}^{s}+c_{i_{1}, i_{2}, \cdots, i_{k-1}, 2}^{s}\right)}
$$

for each $\tau=i_{1}, i_{2}, \cdots, i_{k-1}, i_{k}$, where $i_{j} \in\{1,2\}$.

Example 4. If $\left\{s_{\sigma \mid k}\right\}$ converges so fast to s for each $\sigma$ (To be precise, assume $\sum_{k=0}^{\infty}\left|\log \left(c_{\sigma \mid k, 1}^{s}+c_{\sigma \mid k, 2}^{s}\right)\right|$ converges for each $\sigma$ and their limits are bounded. $)$, the deranged Cantor set has positive and finite s-dimensional Hausdorff and packing measure. 
Proof. The example can be produced by using arguments similar to those used in Example 3.

Remark. In this paper, we only considered a construction of left subinterval $I_{\tau, 1}$ and the right subinterval $I_{\tau, 2}$ of $I_{\tau}$ deleting a middle open subinterval of $I_{\tau}$ inductively for each $\tau \in\{1,2\}^{n}$, where $n=0,1,2, \cdots$. However we could obtain the same result in a deranged Cantor set from the construction that the left and right subintervals are just contained in the fundamental interval with its contracting ratios uniformly bounded away from 0 and separated by the gap ratios also uniformly bounded away from 0 . In this case we need the same arguments of this paper to show it.

Acknowledgments. This work was partly done during visiting the University of North Texas in the summer in 1997. I wish to thank Professor R. Daniel Mauldin for helping to find this topic.

\section{References}

[1] I. S. Baek, Dimensions of the perturbed Cantor set, Real Analysis Exchange, 19 (1994), 269-273.

[2] I. S. Baek, On deranged Cantor sets, Kyungpook Math. Journal, too appear.

[3] K. J. Falconer, Fractal geometry (John Wiley and Sons. 1990).

[4] K. J. Falconer, Techniques in fractal geometry (John Wiley and Sons. 1997).

[5] C. Tricot, Two definitions of fractional dimension, Math. Proc. Camb. Phil. Soc., 91 (1982), 57-74. 